\title{
Children with SARS-CoV-2 infection during an epidemic in China (outside of Hubei province)
}

\author{
Feifan Xiao $^{1,2,3 \#}$, Bin Chen ${ }^{1 \#}$, Tiantian Xiao ${ }^{1}$, Shoo K. Lee ${ }^{4,5}$, Kai Yan ${ }^{1}$, Liyuan Hu ${ }^{1}$ \\ ${ }^{1}$ Department of Neonatology, ${ }^{2}$ Center for Molecular Medicine, Children's Hospital of Fudan University, Shanghai, China; ${ }^{3}$ Institutes of Biomedical \\ Sciences, Fudan University, Shanghai, China; ${ }^{4}$ Departments of Pediatrics, Obstetrics and Gynecology, and Public Health, University of Toronto, \\ Toronto, ON, Canada; ${ }^{5}$ Department of Pediatrics, Mount Sinai Hospital, Toronto, ON, Canada \\ Contributions: (I) Conception and design: SK Lee, K Yan, L Hu; (II) Administrative support: SK Lee; K Yan, L Hu; (III) Provision of study materials \\ or patients: None; (IV) Collection and assembly of data: F Xiao, B Chen, T Xiao; (V) Data analysis and interpretation: F Xiao, B Chen, T Xiao; (VI) \\ Manuscript writing: All authors; (VII) Final approval of manuscript: All authors. \\ \#These authors contributed equally to this work. \\ Correspondence to: Liyuan Hu, MD. Department of Neonatology, Children's Hospital of Fudan University, 399 Wanyuan Road, Shanghai, China. \\ Email: nowadays921@126.com; Kai Yan, MD. Department of Neonatology, Children's Hospital of Fudan University, 399 Wanyuan Road, Shanghai, \\ China. Email: fhyankai@163.com; Shoo K. Lee, MBBS, FRCPC, PhD, DHC, OC. Departments of Pediatrics, Obstetrics and Gynecology, and \\ Public Health, University of Toronto, Toronto, ON, Canada; Department of Pediatrics, Mount Sinai Hospital, 600 University Avenue, Room 19- \\ 231M, Toronto, Ontario M5G 1X5, Canada. Email: shoo.lee@sinaihealth.ca.
}

Background: Limited studies have reported the clinical and epidemiological characteristics of children infected with severe acute respiratory syndrome coronavirus 2 (SARS-CoV-2). This study aimed to provide the epidemiological characteristics of children with COVID-19 throughout China (outside of Hubei Province) based on public data.

Methods: This was an observational, cross-sectional study. We included a total of 279 diagnosed children based on the data of children infected with SARS-CoV-2 reported by the Health Commission in each province, autonomous region, municipality, or special administrative region in China (outside of Hubei Province) from January 24, 2020 to February 16, 2020.

Results: The number of newborns ( 0 days $\leq$ age $\leq 28$ days), infants ( 28 days $<$ age $\leq 1$ year), children ( 1 year $<$ age $\leq 5$ years), and children (5 years < age $\leq 18$ years) accounted for $0.7 \%, 6.5 \%, 23.7 \%$, and $69.2 \%$ of the total number of infected children, respectively. We found that from January 23, 2020, to January 31, 2020, infected children mainly came from Wuhan, China. After February 3, 2020, family clustering transmission became the main mode of transmission.

Conclusions: Family clustering transmission is currently the main model of transmission in children. Considering the mild symptoms in infected children, the possibility that children may be a source of the transmission should not be ignored.

Keywords: Severe acute respiratory syndrome coronavirus 2 (SARS-CoV-2); COVID-19; children

Submitted Mar 26, 2020. Accepted for publication Jun 15, 2020.

doi: $10.21037 / \mathrm{atm}-20-2908$

View this article at: http://dx.doi.org/10.21037/atm-20-2908

\section{Introduction}

The severe acute respiratory syndrome coronavirus 2 (SARS-CoV-2) disease (COVID-19) has been causing a major public health concern (1). Due to the high transmission efficiency of the SARS-CoV-2, the World
Health Organization (WHO) declared on January 30, 2020 that the outbreak of COVID-19 constituted a Public Health Emergency of International Concern. As of May 1, 2020, WHO declared that more than 3,000,000 confirmed cases had been identified worldwide and more than 200,000 
deaths had occurred as a result of the outbreak.

Earlier, on January 10, 2020, a 10-year-old child, who was the first reported child case worldwide, was diagnosed with SARS-CoV-2 infection in Guangzhou (2). With the spread of the SARS-CoV-2 infection, a lot more children have been infected. However, limited studies (3-5) have reported the clinical and epidemiological characteristics of children who have SARS-CoV-2 infection. In this study, we aimed to provide the epidemiological characteristics of children with SARS-CoV-2 infection throughout China (outside of Hubei Province), based on public data. We present the following article in accordance with the STROBE reporting checklist (available at http://dx.doi.org/10.21037/atm-20-2908).

\section{Methods}

\section{Study design and participants}

This study was an observational, cross-sectional study. We included diagnosed children based on data on children infected with SARS-CoV-2 reported by the Health Commission in each province, autonomous region, municipality, or special administrative region in China (outside of Hubei Province) from January 24, 2020 to February 16, 2020. Hubei Province was excluded, because detailed infection data were not available. This study was approved by the Ethics Commission of Children's Hospital of Fudan University \{CHFudan [No. (2020)31]\}. The data were collected from public websites, and the requirement for informed consent was not necessary.

\section{Data collection}

As 31 provinces (autonomous regions or municipalities) in China have successively launched level I responses to serious health emergencies, the release of information has become more transparent. The health committees of all provinces or cities at the prefecture level publicize, the details of epidemiological investigations of confirmed patients every day. Based on those public data, we collected patient information, including gender, age, date of diagnosis, province, address, places visited before the disease onset, and history of close contact with diagnosed patients. We obtained the data of children infected from the websites of the health committees of provinces and prefecturelevel cities in China. The movement track information of the infected children before their diagnosis, and detailed epidemiological information were obtained from daily updates of the provincial official news media platforms.

\section{Laboratory testing}

After patient samples were collected from the designated hospitals, the local Center for Disease Control and Prevention (CDC) staff would send the samples to the CDC SARS-CoV-2 testing laboratory for etiological testing as soon as possible. According to the requirements of the National Health Commission of China, the collection, transportation, storage, and testing of SARS-CoV-2 screening samples should be managed in accordance with the second class of highly pathogenic microorganisms and the regulations on Biosafety Management of Pathogenic Microbiology Laboratories and the Transportation of Highly Pathogenic Microorganisms or Samples that can infect Human Beings.

\section{Diagnosis}

The diagnosis of children infected with SARS-CoV-2 was based on new guidelines for the prevention and control of coronavirus pneumonia issued by the National Health Commission of China. Under these guidelines, patients with a positive RT-PCR nucleic acid test result were considered as confirmed cases. All confirmed diagnoses had clear nucleic acid test results.

\section{Statistical analysis}

Stata 15.0 was used for statistical analysis. Continuous variables were expressed by median and range, while categorical variables were expressed by number and percentage. A univariate analysis was conducted using $\chi^{2}$ tests or Fisher's exact test for categorical data. In the analysis of spatial distribution, we combined the numbers of infected children in each province with reference to the seven geographical regions of China (South China, East China, Central China, North China, Northeast, Northwest, and Southwest). A probability $<0.05$ was considered statistically significant. Data from Hubei Province were excluded from the statistical analysis.

\section{Results}

\section{Demographic information and epidemiological characteristics}

A total of 279 children infected with SARS-CoV-2 were included in this study (Table 1). The numbers of newborns ( 0 days $\leq$ age $\leq 28$ days), infants ( 28 days $<$ age $\leq 1$ year), 
Table 1 Characteristics of included children cases

\begin{tabular}{|c|c|c|c|c|c|c|}
\hline Characteristics & Total & $\begin{array}{c}\text { Newborn }(0 \leq \text { age } \\
\leq 28 \text { days })\end{array}$ & $\begin{array}{l}\text { Infant (28 days < } \\
\text { age } \leq 1 \text { year old })\end{array}$ & $\begin{array}{c}\text { Children }(1 \text { year old < } \\
\text { age } \leq 5 \text { years old })\end{array}$ & $\begin{array}{c}\text { Children }(5 \text { years old }< \\
\text { age } \leq 18 \text { years old })\end{array}$ & $\mathrm{P}$ \\
\hline Male (\%) & $148(53.1)$ & $1(50.0)$ & $8(44.4)$ & $29(43.9)$ & $110(57.0)$ & $>0.05$ \\
\hline Age, median [range] & 9 [0.01-18] & $0.03[0.01-0.05]$ & $0.875[0.08-1]$ & $3[1.08-5]$ & $12[6-18]$ & \\
\hline Age (\%) & $279(100.0)$ & $2(0.7)$ & $18(6.5)$ & $66(23.7)$ & $193(69.2)$ & \\
\hline From the epidemic area (\%) & $141(50.5)$ & $2(100.0)$ & $12(66.7)$ & $31(47.0)$ & $96(49.7)$ & $>0.05$ \\
\hline Family clustering (\%) & $138(49.5)$ & 0 & $6(33.3)$ & $35(53.0)$ & $97(50.3)$ & \\
\hline Asymptomatic (\%) & $38(13.6)$ & $2(100.0)$ & $1(5.6)$ & $6(9.1)$ & $29(15.0)$ & $>0.05$ \\
\hline
\end{tabular}

children ( 1 year < age $\leq 5$ years), and children ( 5 years < age $\leq 18$ years) accounted for $0.7 \%, 6.5 \%, 23.7 \%$, and $69.2 \%$ of the total number of infected children, respectively. The number of infected children under the age of five accounted for nearly one third of all infected children. The median incubation period for children was six days, with a minimum of one day and a maximum of 24 days. There was no significant difference in latency among the four age groups $(\mathrm{P}>0.05)$. Children from the epidemic area accounted for $50.5 \%$ of the infected children, and $49.5 \%$ of the children had a history of family aggregation or close contact with diagnosed patients. Further, $13.6 \%$ of the children had no clinical symptoms (such as fever, sore throat, cough, or diarrhea). The median time of children who got virologic diagnosis was 2 days (from the symptom onset or the start of medical observation). Among the four different age groups, there were no statistical differences in sex, incubation period, mode of transmission, number of asymptomatic infections, or days of diagnosis. Only one child became seriously ill and no child deaths have been reported.

\section{Cases of a long incubation period and asymptomatic infection}

\section{Case 1}

In Rizhao City, Shandong Province, two asymptomatic children with coronavirus ( 5 and 11 years old) in a single family were reported on February 15, 2020. The children's grandfather was diagnosed with SARS-CoV-2 infection on January 20, 2020. Then the family members were quarantined and throat swabs of the children, and their parents showed negative results for SARS-CoV-2 infection. However, the children's father presented with a fever and a cough and was diagnosed with COVID-19 on February 14, 2020. The next day, the two children and their mother were diagnosed with asymptomatic coronavirus infection.

\section{Case 2}

A 4-year-old girl from Wuhan. She and her family arrived in Sanya City, Hainan Province by plane from Wuhan City, Hubei Province on December 12, 2019, and then went to Lingshui City by car. They had no fever, cough, or any other symptoms. On January 8 , one of the girl's relatives arrived in Lingshui City from Wuhan City and was diagnosed SARS-CoV-2 infection on February 1. Around January 26, the girl's grandmother developed a fever and was diagnosed on January 31 . The girl was diagnosed through a nucleic acid test on February 1, during the period of medical isolation.

\section{Spatial distribution of SARS-CoV-2 infection in children}

The results showed that the three regions with the largest numbers of children infected were South China (25.4\%), East China (24.7\%), and Central China (23.7\%). The total number of infected children in these three regions accounts for about two thirds of the infected children in the country. The number of infected children in Northwest and Northeast China was relatively small (Figure 1A). 

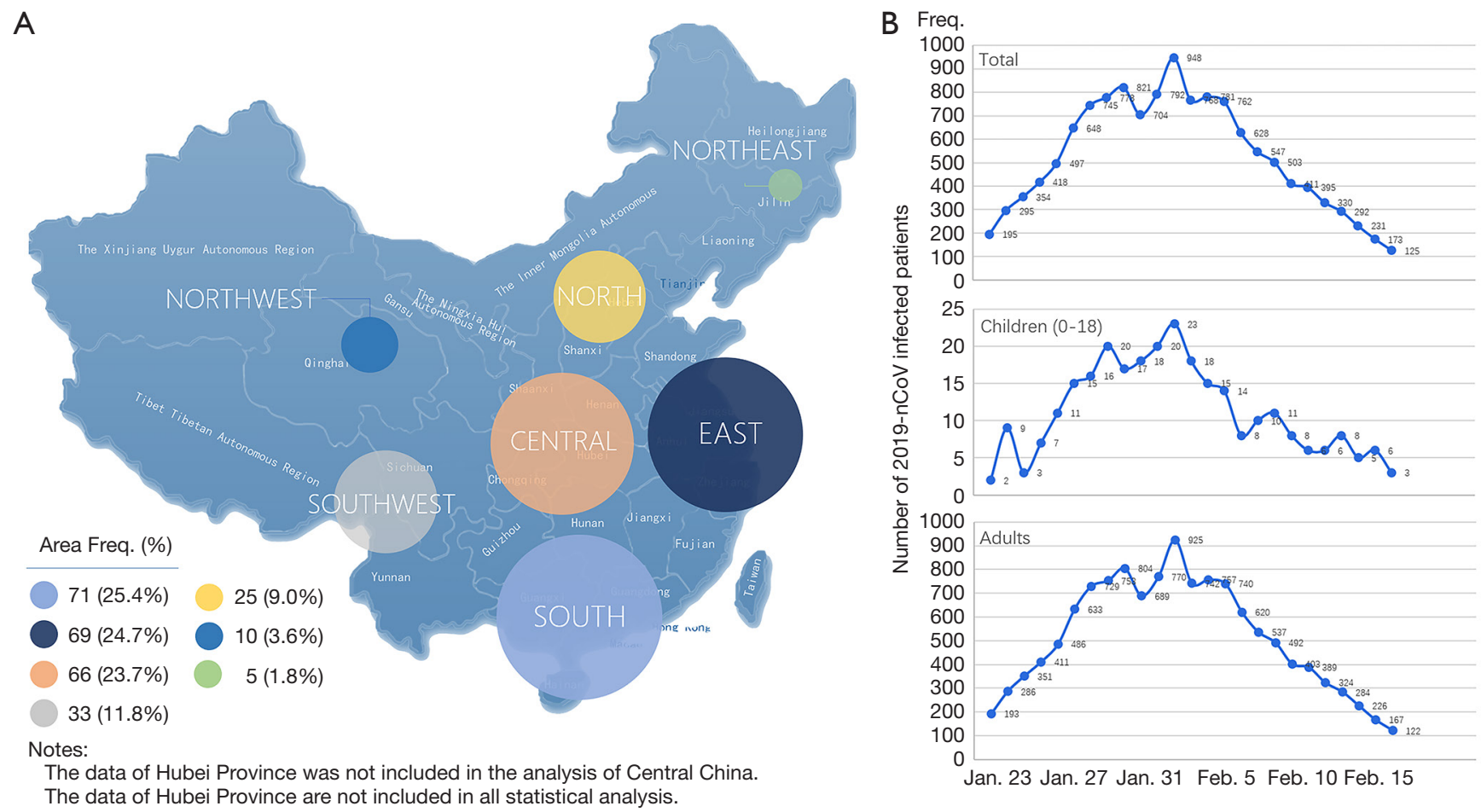

Figure 1 Spatial and temporal distribution of children infected with SARS-CoV-2. (A) Spatial distribution of SARS-CoV-2 infection in children; (B) time trend of SARS-CoV-2 infection in children and adults.

\section{Time trend of SARS-CoV-2 infection in children and adults}

Twelve days after Hubei Province implemented the lockdown on January 23, 2020, the number of SARS-CoV-2 infections in China (except Hubei Province) reached its peak on February 3, 2020. The incidence time trend of daily new infections in children was similar to that in adults, as shown in Figure $1 B$.

Figure 2 shows that, at the early stage of the epidemic, the increase in the number of infections was mainly related to the export of mobility in the epidemic area (Hubei Province). As time progressed, after exceeding the average incubation period (January 31, 2020), the number of imported cases in the epidemic area began to decrease gradually, while the number of cases due to close family contact began to increase.

\section{Discussion}

Many Chinese provinces have reported cases of children infected with SARS-CoV-2. The youngest infected child was a neonate that was infected 30 hours after its birth (6). As of May 1, 2020, 84,385 cases had been confirmed in
China. With the increase in the size of the confirmed population, the number of infected children has also increased. At present, the risk of death after infection is much lower among children than it is among adults. Previous studies $(7,8)$ found higher percentages of infection in men than in women, but our analysis showed no gender difference in children. We found that two thirds of infected children were over 5 years old, while only one third of all the infected children were under 5 years old. However, newborns, infants, school-aged children, and teenagers were all susceptible to the virus.

Fever and cough were the most common symptoms in adults infected with SARS-CoV-2 $(9,10)$. Wei and colleagues (11) reported that only four of nine infected infants presented with fever. All infected infants presented with mild clinical symptoms. Zeng et al. also reported similar results in three neonates infected with SARSCoV-2 (6). In our study, we found that most children showed mild clinical symptoms after infection, $13.6 \%$ of the children had no clinical symptoms after infection, and some asymptomatic infections were identified after virologic screening during the quarantine. Thus, one concern is that a lack of timely screening might result in missed diagnoses 


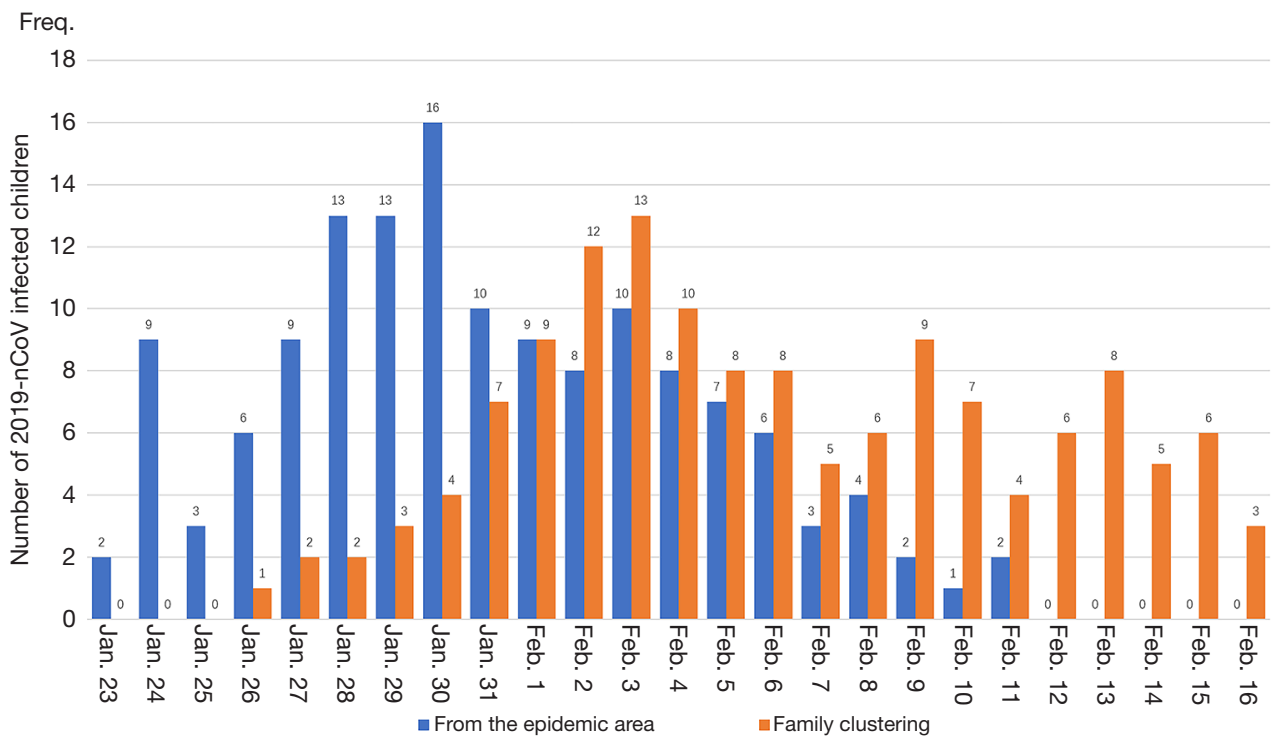

Figure 2 Changes in transmission mode in children at different time points.

for these children and a potentially wider spread of the SARS-CoV-2. Researchers have found that SARS-CoV-2 replicates not only in the lungs but also in the nose, throat, and gut, and even persons with mild symptoms can transmit the virus (12). Currently, no evidence supports the notion that asymptomatic individuals cannot transmit the virus to others. A study (13) indicated that asymptomatic MERS$\mathrm{CoV}$ cases is about $9.8 \%$. If a similar phenomenon is confirmed for COVID-19, the prevention and control of SARS-CoV-2 infection will be more difficult. In addition, a recent study (14) reported that, compared with SARS$\mathrm{CoV}$, the affinity between spike protein and Angiotensinconverting enzyme 2 receptor in SARS-CoV-2 has increased 10 to 20 fold, suggesting that SARS-CoV-2 has a stronger transmission ability.

Based on our analysis, we found that infected children were mainly from Wuhan, China from January 23, 2020 to January 31, 2020. However, after February 3, 2020, family clustering transmission became the main mode of transmission. As a type of coronavirus, SARS-CoV-2 is transmitted mainly via droplet transmission and contact transmission. Although Chen and colleagues (15) concluded that SARS-CoV-2 cannot be vertically transmitted in late pregnancy, the possibility of vertical transmission should not be ignored because the number of related studies is limited. Models of transmission in children should also be explored further.

There are several difficulties in preventing SARS-CoV-2 infections in children currently. First, infected children tend to present with mild clinical symptoms without the classic phenotype of lung pneumonia, which renders infected children difficult to identify. Second, children can be a source of transmission during the viral incubation period. Our analysis found that 15 infected children had an incubation period of more than 14 days, while $13.6 \%$ of the children had no symptoms before the diagnosis. Indeed, it is difficult for investigators to collect information about the symptoms of very young children because they cannot accurately express their symptoms. Thus, we should be aware that children in the early stages of infection may become sources of transmission. Guan and colleagues (16) indicated that the longest incubation period was 24 days, but they did not provide more detailed information. In the two family clustering cases that we reported, the infected children experienced both very long incubation periods and no clinical symptoms. A new prediction model will be needed to consider the differences in patient latency and the spread of the virus from asymptomatic patients. These two factors will have significant effects on the construction of a prediction model for the epidemic.

The best safety measure for children is still prevention (17). For those with early asymptomatic infections, effective remedial interventions may be needed, such as a routine nucleic acid test. If the virus continues to be transmitted from person to person, the epidemic may be difficult to stop. In addition, though scientists are now scrambling to 
develop new diagnostic technologies (18), it remains unclear how long the antibodies can be maintained in patients who have been cured. Thus, COVID-19 tests (nucleic acid test and antibody test) and isolation are necessary for close contacts of people infected with SARS-CoV-2.

The present study has several limitations that should be addressed. First, although more than 60,000 individuals infected with SARS-CoV-2 were reported in Hubei Province, we excluded data from this region because detailed information was not available. Second, as we were conservative during data collection, cases lacking important demographic or epidemiological information (such as gender, age, or mode of transmission) were excluded from the analysis. Finally, the exact exposures leading to the positive tests may not be known. Further studies should be conducted in the future.

\section{Conclusions}

In summary, the results of our study suggest that family clustering transmission is currently the main mode of SARS-CoV-2 transmission among children. Considering that infected children may experience mild symptoms, the possibility that children may be a source of transmission should not be ignored.

\section{Acknowledgments}

Funding: None.

\section{Footnote}

Reporting Checklist: The authors have completed the STROBE reporting checklist. Available at http://dx.doi. org/10.21037/atm-20-2908

Data Sharing Statement: Available at http://dx.doi. org/10.21037/atm-20-2908

Conflicts of Interest: All authors have completed the ICMJE uniform disclosure form (available at http://dx.doi. org/10.21037/atm-20-2908). The authors have no conflicts of interest to declare.

Ethical Statement: The authors are accountable for all aspects of the work in ensuring that questions related to the accuracy or integrity of any part of the work are appropriately investigated and resolved. The study was conducted in accordance with the Declaration of Helsinki (as revised in 2013). This study was approved by the Ethics Commission of Children's Hospital of Fudan University \{CHFudan [No. (2020)31]\}. The data were collected from public websites, and the requirement for informed consent was not necessary.

Open Access Statement: This is an Open Access article distributed in accordance with the Creative Commons Attribution-NonCommercial-NoDerivs 4.0 International License (CC BY-NC-ND 4.0), which permits the noncommercial replication and distribution of the article with the strict proviso that no changes or edits are made and the original work is properly cited (including links to both the formal publication through the relevant DOI and the license). See: https://creativecommons.org/licenses/by-nc-nd/4.0/.

\section{References}

1. Huang C, Wang Y, Li X, et al. Clinical features of patients infected with 2019 novel coronavirus in Wuhan, China. Lancet 2020;395:497-506.

2. Chan JF, Yuan S, Kok KH, et al. A familial cluster of pneumonia associated with the 2019 novel coronavirus indicating person-to-person transmission: a study of a family cluster. Lancet 2020;395:514-23.

3. Zhu H, Wang L, Fang C, et al. Clinical analysis of 10 neonates born to mothers with 2019-nCoV pneumonia. Transl Pediatr 2020;9:51-60.

4. Cai J, Xu J, Lin D, et al. A Case Series of children with 2019 novel coronavirus infection: clinical and epidemiological features. Clin Infect Dis 2020. [Epub ahead of print].

5. Wang D, Ju XL, Xie F, et al. Clinical analysis of 31 cases of 2019 novel coronavirus infection in children from six provinces (autonomous region) of northern China. Zhonghua Er Ke Za Zhi 2020;58:269-74.

6. Zeng L, Xia S, Yuan W, et al. Neonatal Early-Onset Infection With SARS-CoV-2 in 33 Neonates Born to Mothers With COVID-19 in Wuhan, China. JAMA Pediatr 2020;174:722-5.

7. Wang D, Hu B, Hu C, et al. Clinical Characteristics of 138 Hospitalized Patients With 2019 Novel CoronavirusInfected Pneumonia in Wuhan, China. JAMA 2020;323:1061-9.

8. Chen N, Zhou M, Dong X, et al. Epidemiological and clinical characteristics of 99 cases of 2019 novel coronavirus pneumonia in Wuhan, China: a descriptive 
study. Lancet 2020;395:507-13.

9. Zheng F, Tang W, Li H, et al. Clinical characteristics of 161 cases of corona virus disease 2019 (COVID-19) in Changsha. Eur Rev Med Pharmacol Sci 2020;24:3404-10.

10. Yu X, Sun X, Cui P, et al. Epidemiological and Clinical Characteristics of 333 Confirmed Cases with Coronavirus Disease 2019 in Shanghai, China. Transbound Emerg Dis 2020;67:1697-707.

11. Wei M, Yuan J, Liu Y, et al. Novel Coronavirus Infection in Hospitalized Infants Under 1 Year of Age in China. JAMA 2020;323:1313-4.

12. Wölfel R, Corman VM, Guggemos W, et al. Virological assessment of hospitalized patients with COVID-2019. Nature 2020;581:465-9.

13. Al-Tawfiq JA, Gautret P. Asymptomatic Middle East Respiratory Syndrome Coronavirus (MERS-CoV) infection: Extent and implications for infection control: A

Cite this article as: Xiao F, Chen B, Xiao T, Lee SK, Yan K, $\mathrm{Hu}$ L. Children with SARS-CoV-2 infection during an epidemic in China (outside of Hubei province). Ann Transl Med 2020;8(14):849. doi: 10.21037/atm-20-2908 systematic review. Travel Med Infect Dis 2019;27:27-32.

14. Wrapp D, Wang N, Corbett KS, et al. Cryo-EM structure of the 2019-nCoV spike in the prefusion conformation. Science 2020;367:1260-3.

15. Chen H, Guo J, Wang C, et al. Clinical characteristics and intrauterine vertical transmission potential of COVID-19 infection in nine pregnant women: a retrospective review of medical records. Lancet 2020;395:809-15.

16. Guan WJ, Ni ZY, Hu Y, et al. Clinical Characteristics of Coronavirus Disease 2019 in China. N Engl J Med 2020;382:1708-20.

17. Wang L, Shi Y, Xiao T, et al. Chinese expert consensus on the perinatal and neonatal management for the prevention and control of the 2019 novel coronavirus infection (First edition). Ann Transl Med 2020;8:47.

18. Cohen J, Kupferschmidt K. Labs scramble to produce new coronavirus diagnostics. Science 2020;367:727. 